Infectious medicine, virology

\title{
Seroconversion and dynamics of the anti-SARS-CoV-2 antibody response related to a hospital COVID-19 outbreak among pediatric oncology patients
}

\author{
Nikolay Mayanskiy $\mathbb{1}^{1} \cdot$ Polina Luchkina ${ }^{1} \cdot$ Natalia Fedorova $^{1} \cdot$ Yuri Lebedin $\mathbb{D}^{2} \cdot$ Natalia Ponomareva $^{1}$
}

Received: 15 April 2021 / Revised: 29 April 2021 / Accepted: 5 May 2021 / Published online: 18 May 2021

(c) The Author(s), under exclusive licence to Springer Nature Limited 2021

\section{To the Editor:}

Two recent reports published in Leukemia have described the antibody response to SARS-CoV-2 infection in adult patients with chronic lymphocytic leukemia (CLL) [1] and acute leukemia [2] in conjunction with PCR-confirmed COVID-19. These studies have demonstrated that $14 / 21$ (67\%) of the examined CLL patients and 7/8 (88\%) of the examined acute leukemia patients produced anti-SARSCoV-2 IgG antibodies. Importantly, 6 out of 7 IgG-positive acute leukemia patients developed virus-neutralizing antibodies [2], assuming a protective immune response.

Here we describe the anti-SARS-CoV-2 antibody response in a cohort of pediatric oncology patients with PCR-confirmed COVID-19, who became infected during an outbreak at the hemato-oncology department of the Russian children clinical hospital, Pirogov Russian National Research Medical University, at the end of April, 2020. This study enrolled 18 patients with neoplasms (16 patients with acute lymphoblastic leukemia (ALL) in remission and two patients with a brain tumor) receiving multidrug chemotherapy. The median age was 8.2 years (IQR 7.8). All patients with ALL received consolidation chemotherapy based on the local protocol ALL-MB-2015, which included daunorubicin, L-asparaginase, and methotrexate. All patients were tested SARS-CoV-2 PCR-positive on April

\footnotetext{
Supplementary information The online version contains supplementary material available at https://doi.org/10.1038/s41375021-01288-0.

Nikolay Mayanskiy

mayanskiy.nikolay@gmail.com

1 Russian Children Clinical Hospital, Pirogov Russian National Research Medical University, Moscow, Russia

2 XEMA Company, Moscow, Russia
}

28 or 29, 2020. Clinical characteristics are shown in Supplementary Table 1 . The majority of patients demonstrated a mild course of COVID-19 with minimal symptoms. Fever was the most common symptom (14/18); anosmia was reported in 4 (22\%) patients. Among 12 patients with a lung CT scan performed, in $6(50 \%)$ no lung involvement was found, in $5(42 \%)$ and $1(8 \%)$ lung involvement of CT grade 1 and 2, respectively, was observed (Supplementary Table 1).

In total, we collected 113 residual serum specimens from these patients at different time points and detected serum IgM and IgG against the receptor binding domain (RBD) of the $\mathrm{S}$ protein (anti-RBD) and the nucleocapsid protein (antiN) of SARS-CoV-2 (Fig. 1A, Supplementary Table 2) using ELISA kits developed by XEMA Company (Moscow, Russia) (see Supplementary information). In four patients, specimens obtained prior to PCR testing were available, which contained no detectable anti-SARS-CoV-2 antibodies. Seroconversion was observed in $92 \%$ patients by week 3 and in $100 \%$ patients by week 6 post-exposure (Fig. 1A, Supplementary Table 2). The seropositive rate was maintained at around $80 \%$ for at least three consecutive weeks declining to $54 \%$ by week 18 post-exposure (Fig. 1A, Supplementary Table 2).

Anti-RBD IgG were the most prevalent antibodies detected in 92\% patients by week 6 (Fig. 1A, Supplementary Table 2). The highest seroprevalence rate for anti-N IgG was around $70-75 \%$. The rate of anti-RBD IgM was $23-44 \%$ by weeks 3 to 4 ; IgM antibodies vanished at the end of the 9-week follow-up period. Anti-N IgM were detected in two patients (in one patient simultaneously with anti-RBD IgM).

To assess the anti-SARS-CoV-2 antibody profile distribution at the specimen level, we selected virus-specific Ig-positive serum specimens, where both anti-SARS-CoV-2 $\operatorname{IgG}$ and $\operatorname{IgM}$ antibodies were measured $(n=85)$. The majority of specimens $(71 / 85 ; 84 \%)$ contained anti-RBD 
Fig. 1 Dynamics and magnitude of the anti-SARSCoV-2 antibody response in pediatric oncology patients, by week after a positive PCR test for SARS-CoV-2. A The dynamics of the anti-SARS$\mathrm{CoV}-2$ IgG and IgM antibody seroprevalence rate was examined in 133 serum specimens obtained at the indicated time points from 18 pediatric oncology patients after a SARS-CoV-2 PCR-positive test. At week 18 , only IgG antibodies were analyzed. The level of anti-RBD (B) and anti-N (C) IgG antibodies are depicted as median $(\mathrm{Me})$ positivity indexes (PI) representing the ratio between the specimen and the internal cut-off control optical density in the ELISA assay. Error bars indicate interquartile ranges (IQR). Dashed lines on (B and $\mathbf{C}$ ) show the positivity threshold. The actual number of specimens analyzed by time points is given in Supplementary Table 2.
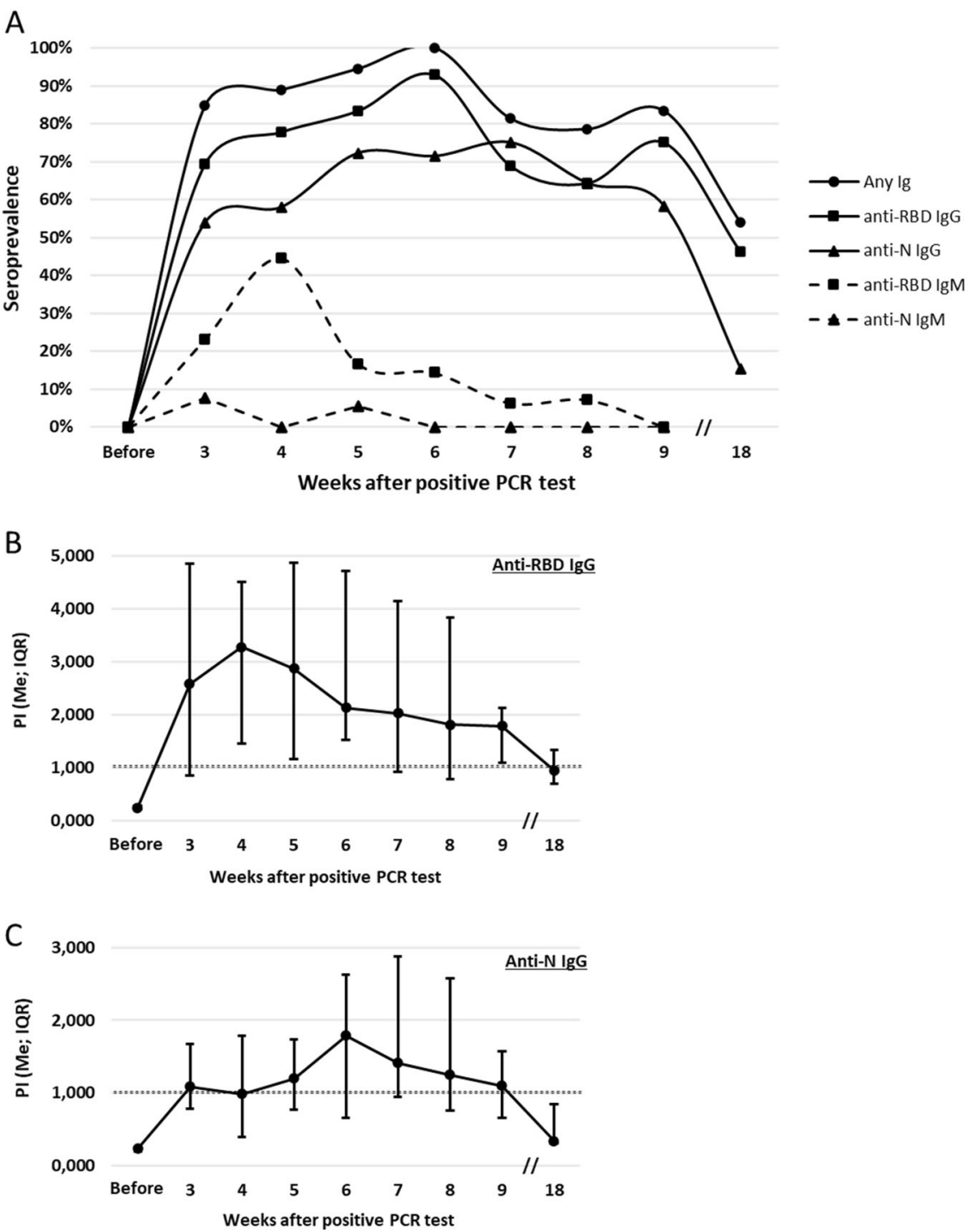

IgG antibodies alone or in combination with anti-N $\operatorname{IgG}$ and/or anti-RBD IgM (Supplementary Table 3). In 8/85 (9\%) specimens, exclusively anti-N IgG was present. AntiSARS-CoV-2 IgM was detected in 15/85 (18\%) virusspecific Ig-positive specimens; all IgM-positive specimens concurrently contained virus-specific IgG antibodies, except one specimen, where only anti-RBD IgM was detected (Supplementary Table 3).

Next, we analyzed the magnitude of the IgG antibody response using positivity index (PI) values calculated as the ratio between the specimen and the internal cut-off control optical density, which reflected the antibody level (Fig. 1B, C). The level of anti-RBD IgG increased up to a median PI value of 3.27 (IQR 3.04) over weeks 3-4, and plateaued at a median PI value around 2.0 by week 6 persisting at that level at least until week 9. At the last flow-up time point (week 18), a median PI value declined to 0.95 (IQR 0.64). The level of anti-N IgG demonstrated a slightly different dynamics with a delayed peak PI value of 1.79 (IQR 1.96) by week 6 (Fig. 1B, C).

Our findings addressed several important issues. We demonstrated that pediatric patients with hematological neoplasia or solid tumors responded to a SARS-CoV-2 infection producing antibodies of different isotypes against the RBD and $\mathrm{N}$ target antigens of the virus. Interestingly, a report has suggested that children may have a distinct antibody response to SARS-CoV-2 compared to adults and generate predominantly anti-spike antibodies, but not anti-N [3], which we did not observe in the present study. All examined patients demonstrated seroconversion, which persisted in more than a half of patients for at least 18 weeks. The functionality and longevity of the immune response against SARS-CoV-2 in such patients warrant further elaboration. As was mentioned above, adult acute leukemia patients were capable of developing neutralizing anti-SARS-CoV-2 antibodies [2]. In our study, the majority 
of patients demonstrated the presence of anti-spike (antiRBD) antibodies, which titers appear to closely correlate with the neutralizing capacity [4]. Moreover, a recent metaanalysis has demonstrated that pediatric patients with COVID-19 suffering from hematological neoplasia or solid tumors were not at an increased risk of unfavorable outcomes [5]. Taken together, these data may indicate that pediatric patients with malignancies are unlikely to be more vulnerable to COVID-19 comparing to other children, as had been noticed earlier [6]. However, the situation may be different in hospitalized adults with hematological neoplasia and accompanying COVID-19, who have demonstrated more severe disease and a higher case fatality rate [7].

Our results indicated that although the majority of specimens contained anti-RBD IgG antibodies, among the antiSARS-CoV-2 IgG-positive specimens there were $13 \%$ where exclusively anti-N IgG occurred. This finding suggested that the detection of antibodies to both targets would increase the sensitivity of serological analysis.

In conclusion, further study of the immune response to SARS-CoV-2 infection in children with cancer is critical for providing medical care and implementing optimal protection in this patient group in the context of the COVID-19 pandemic [8].

Acknowledgements The RNA extraction and RT-PCR were performed using the equipment of Center for Precision Genome Editing and Genetic Technologies for Biomedicine, Pirogov Russian National Research Medical University, Moscow, Russia (grant 075-15-20191789).

\section{Compliance with ethical standards}

Conflict of interest YL is an employee of XEMA Company. All other authors have no competing interests
Publisher's note Springer Nature remains neutral with regard to jurisdictional claims in published maps and institutional affiliations.

\section{References}

1. Roeker LE, Knorr DA, Pessin MS, Ramanathan LV, Thompson MC, Leslie LA, et al. Anti-SARS-CoV-2 antibody response in patients with chronic lymphocytic leukemia. Leukemia. 2020;34:3047-3049. https://doi.org/10.1038/s41375-020-01030-2.

2. O'Nions J, Muir L, Zheng J, Rees-Spear C, Rosa A, Roustan C, et al. SARS-CoV-2 antibody responses in patients with acute leukaemia. Leukemia. 2021;35:289-292. https://doi.org/10.1038/ s41375-020-01103-2.

3. Weisberg SP, Connors TJ, Zhu Y, Baldwin MR, Lin WH, Wontakal S, et al. Distinct antibody responses to SARS-CoV-2 in children and adults across the COVID-19 clinical spectrum. Nat Immunol. 2021;22:25-31. https://doi.org/10.1038/s41590-02000826-9.

4. Wu J, Liang B, Chen C, Wang H, Fang Y, Shen S, et al. SARS-CoV2 infection induces sustained humoral immune responses in convalescent patients following symptomatic COVID-19. Nat Commun. 2021;12:1813 https://doi.org/10.1038/s41467-021-22034-1.

5. Dorantes-Acosta E, Ávila-Montiel D, Klünder-Klünder M, JuárezVillegas L, Márquez-González H. Survival and complications in pediatric patients with cancer and COVID-19: a meta-analysis. Front Oncol. 2021;10:608282 https://doi.org/10.3389/fonc.2020. 608282.

6. Boulad F, Kamboj M, Bouvier N, Mauguen A, Kung AL. COVID-19 in children with cancer in New York city. JAMA Oncol. 2020;6:1459-1460. https://doi.org/10.1001/jamaoncol. 2020.2028

7. He W, Chen L, Chen L, Yuan G, Fang Y, Chen W, et al. COVID19 in persons with haematological cancers. Leukemia 2020;34:1637-1645.

8. von Lilienfeld-Toal M, Vehreschild JJ, Cornely O, Pagano L, Compagno F, EHA Infectious Disease Scientific Working Group, Hirsch HH. Frequently asked questions regarding SARS-CoV-2 in cancer patients-recommendations for clinicians caring for patients with malignant diseases. Leukemia. 2020;34:1487-1494. 